\title{
Leitmotive und Storylines der Energiewende
}

\author{
Wie Stakeholder-Positionen systematisch \\ in Transformationspfade integriert werden können
}

Ricarda Schmidt-Scheele, Zentrum für interdisziplinäre Risiko- und Innovationsforschung (ZIRIUS), Universität Stuttgart, Seidenstraße 36, 70174 Stuttgart (ricarda.schmidt-scheele@zirius.uni-stuttgart.de) (D) orcid.org/0000-0003-0491-9981

Dierk Bauknecht, Öko-Institut e.V., Institut für angewandte Ökologie (d.bauknecht@oeko.de) (1) orcid.org/0000-0002-5816-0176

Witold-Roger Poganietz, Institut für Technikfolgenabschätzung und Systemanalyse (ITAS), Karlsruher Institut für Technologie (KIT) (witold-roger.poganietz@kit.edu)

Dominik Seebach, Öko-Institut e.V., Institut für angewandte Ökologie (d.seebach@oeko.de)

Christof Timpe, Öko-Institut e. V., Institut für angewandte Ökologie (c.timpe@oeko.de)

Wolfgang Weimer-Jehle, Zentrum für interdisziplinäre Risiko- und Innovationsforschung (ZIRIUS), Universität Stuttgart

(wolfgang.weimer-jehle@zirius.uni-stuttgart.de) (D) orcid.org/0000-0002-2945-7288

Annika Weiss, Institut für Technikfolgenabschätzung und Systemanalyse (ITAS), Karlsruher Institut für Technologie (KIT) (annika.weiss@kit.edu)

Transformationspfade zu einem nachhaltigen Energiesystem beruhen meist auf modellbasierten Szenarien. In den Szenarien müssen gesellschaftliche Prozesse und deren Interaktion mit technologischen, ökonomischen und ökologischen Aspekten betrachtet werden. Dies setzt u. a. eine Integration zentraler Stakeholder-Positionen in die Szenarien voraus. Hierzu präsentieren wir Ansätze aus zwei Forschungsprojekten: Der erste Ansatz identifiziert gesellschaftliche Leitmotive der Energiewende und analysiert, in welchen technisch-ökonomischen Transformationspfaden diese realisiert werden können. Der zweite Ansatz setzt auf eine partizipative Entwicklung von Storylines, um eine verbesserte Legitimation und Kommunikation von Transformationspfaden zu erreichen. Wir diskutieren die Herangehensweisen beider Ansätze, die Positionen von Stakeholdern methodisch zu erfassen und mit technisch-ökonomischen Perspektiven zur Energiesystemtransformation zu verknüpfen.

\section{Guiding motives and storylines of the German energy transition} How to systematically integrate stakeholder positions into energy transformation pathways

Pathways towards sustainable energy transformations are usually premised on model-based scenarios. These scenarios have to incorporate societal dynamics and their interaction with technological, economic, and ecological processes. This also requires the consideration and integration of the stakeholders' positions into the scenarios. For this purpose, we present two different approaches: the first one identifies the stakeholders' guiding motives regarding the German energy

This is an article distributed under the terms of the Creative Commons Attribution License CCBY 4.0 (https://creativecommons.org/licenses/by/4.0/)

https://doi.org/10.14512/tatup.28.3.27

Submitted: 28.06.2019. Peer reviewed. Accepted: 23.10.2019 transition and analyzes how these positions can be realized in technological and economic transformation pathways. In the second approach, storylines are developed in a participatory process with the stakeholders to ensure increased legitimacy and communication of the resulting pathways. For both approaches, we discuss the methodological capture of stakeholder positions and their subsequent integration into energy transformation pathways.

Keywords: energy system transformation, energy scenarios, energy values, stakeholder involvement

\section{Einleitung}

In der Transformationsforschung findet der Ansatz, modell-basierte Energieszenarien mit systematischen Ausarbeitungen gesellschaftlicher und politischer Kontexte zu kombinieren, zunehmende Aufmerksamkeit. Hierzu kann auf eine Reihe von Methoden verwiesen werden (Nakićenović 2000; Weimer-Jehle et al. 2013; Trutnevyte et al. 2014). Wissenschaftlich fundierte Einschätzungen darüber, unter welchen gesellschaftlichen Bedingungen technisch-ökonomische Entwicklungspfade realisiert werden können, stellen einen wichtigen Schritt in Richtung einer Systematisierung von Kontextunsicherheit und -komplexität dar. In diesem Zusammenhang wird von der interdisziplinären Energieforschung gefordert, mögliche Transformationspfade nicht lediglich ex-post hinsichtlich der Positionen von Stakeholdern zu bewerten, sondern ebendiese bereits bei der Konstruktion von Transformationspfaden zu berücksichtigen (Berkhout 2006). 


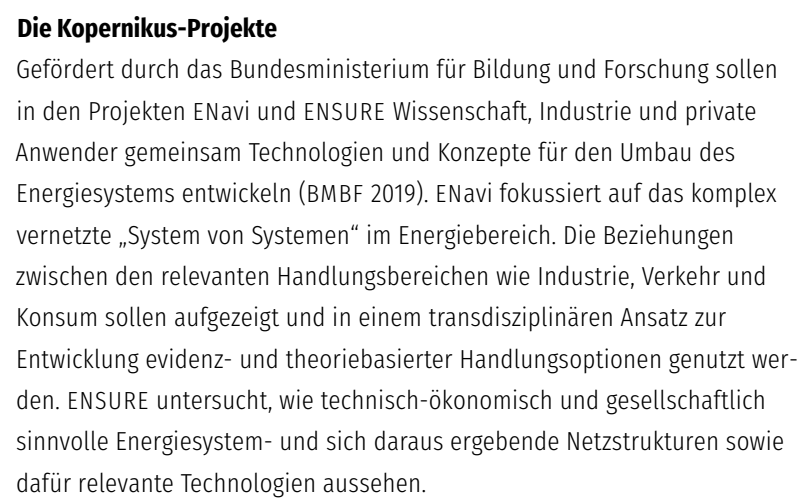

Ein Argument für diese Vorgehensweise ist die daraus resultierende Plausibilität der Pfade. Die Einbindung von Stakeholder-Positionen reflektiert die tatsächliche Vielschichtigkeit soziotechnischer Transformationen. Zudem fördert sie auch die Legitimität und potenzielle Wirksamkeit von Szenarien in der Beratung von Entscheidungsträgern, da kollektive Zukunftsvorstellungen und Visionen als wesentliche Motivation und Orientierung von Akteurshandeln eingeschätzt werden (Heyen et al. 2018, S. 18).

Stakeholder-Positionen umfassen Wissensbestände, die in der Transformationsforschung als Ziel- und Orientierungswissen konzeptualisiert werden (Becker und Jahn 1999). Sie beinhalten grundsätzliche Zielvorstellungen in Form präferierter Maßnahmen oder Technologien (Mielke et al. 2016), aber auch Grundprinzipien zur Ausgestaltung der Energiewende, z. B. eine faire Verteilung von Lasten und Risiken (Cuppen et al. 2016). Im Gegensatz zu den meist qualitativen Positionen generieren Modellierungen wichtiges Systemwissen auf Basis klar definierter technischer und ökonomischer Parameter. Vor diesem Hintergrund widmen wir uns zwei methodischen Herausforderungen der Transformationsforschung: 1) Wie können Stakeholder-Positionen empirisch identifiziert und systematisch aufgearbeitet werden? 2) Wie können die Positionen nicht nur additiv mitberücksichtigt, sondern in das technisch-ökonomische Systemwissen integriert werden? Die Forschungsprojekte ENavi und ENSURE präsentieren hierzu unterschiedliche methodische $\mathrm{Zu}$ gänge. Beide Projekte haben das Ziel, Transformationspfade zu entwickeln, die technisch-ökonomisch machbar sind, aber auch gesellschaftlich akzeptiert werden. Wir vergleichen die Ansätze hinsichtlich der methodischen Herausforderungen und diskutieren, welche Implikationen sich für die Nutzung der Ergebnisse durch Entscheidungsträgern ergeben.

\section{Leitmotive der Energiewende}

Modellierte Transformationspfade verwenden häufig politische Zielvorgaben als Ausgangspunkte, z. B. die Reduktion von Treibhausgasemissionen bis 2050 um 80-95\% gegenüber 1990 .
Diese Vorgaben definieren die Ziele der Transformation, jedoch nicht die möglichen Wege dorthin. Es verbleibt ein Möglichkeitskontinuum zielerfüllender Transformationspfade. Um die Vielzahl der möglichen Pfade vor dem Hintergrund gesellschaftlicher Entwicklungen aufzugliedern und diskutierbar zu machen, werden im Projekt ENavi Leitmotive der Energiewende herangezogen.

\section{Prozessbeschreibung}

Unter Leitmotiven verstehen wir Vorstellungen von Stakeholdern darüber, wie und nach welchen Prinzipien der Transformationsprozess gestaltet werden soll. Leitmotive sind implizit oder explizit in der Gesellschaft vorhanden. Dieser Ansatz basiert auf den Konzepten Leitbilder (de Haan 2001) und Leitorientierungen (Gleich et al. 2010), die beide in der Transformationsforschung genutzt werden, um kollektive Vorstellungen, basierend auf Werten und Weltbildern, gesellschaftlich zu verhandeln. Eine besondere methodische Herausforderung besteht darin, dass sie Zielvorstellungen anschaulich hervorheben, jedoch ,,in aller Regel noch keine Aussagen über die hierfür notwendigen konkreten Maßnahmen zur Erreichung machen" (Giesel 2007, S. 77). Wir verwenden Leitmotive als Analysekonzept. Es nimmt im besonderen Maße einzelne, tiefer liegende Beweggründe für bestimmte Positionen in den Blick (Rosenstiel 2007) und versteht sie als orientierungs- und handlungsleitend für Stakeholder in Bezug auf deren Transformationsentscheidungen.

Die Identifizierung von Leitmotiven im gesellschaftlichen Diskurs erfolgt im Rahmen einer qualitativen Inhaltsanalyse von Primärquellen (de Haan 2001). Ausgewertet werden Positionspapiere zur Energiewende, die eine Vielfalt von Positionen und Interessen beinhalten (u. a. Kommunen, Wirtschafts- und Umweltverbände, Energieerzeuger, Netzbetreiber). In der Dokumentenanalyse werden die Kategorien Wunsch- (was wird als wünschenswertes Ziel erachtet?) und Machbarkeitsprojektionen (was kann und soll in der Zukunft erreicht werden?) genutzt. Zusätzlich wird codiert, welche Rollen und Verantwortlichkeiten unterschiedlichen Akteuren zugeschrieben werden. Das Ergebnis sind verdichtete Beschreibungen der Leitmotive. Insgesamt werden sieben Leitmotive identifiziert. Neben dem in Tab. 1 verkürzt dargestellten exemplarischen Leitmotiv sind dies Energiewende als Generationenprojekt, die minimalinvasive Ener-

\footnotetext{
Energiewende als Chance für die gesellschaftliche Modernisierung

Die notwendigen Umbrüche im Energiesystem werden als Chance gesehen, verfestigte Strukturen aufzubrechen und sie durch eine verstärkte Demokratisierung und Partizipation zu ersetzen. Wie die Energiewende zu gestalten ist, wird kontinuierlich diskutiert und verhandelt werden. Planung und Realisierung von Infrastrukturvorhaben auf lokaler Ebene durch lokale Akteure (Bürgerenergiegesellschaften, Stadtwerke, Kommunen) bieten die Gelegenheit für ein gestärktes bürgerliches Engagement.
}

Tab.1: Exemplarisches Leitmotiv der Energiewende. Quelle: Eigene Darstellung 
giewende, EU in der energiepolitischen Führungsrolle, Deutschland als energiepolitischer Vorreiter in der Welt, Energiewende als Motor für Chancengleichheit zwischen den Regionen der BRD, Energiewende zur Erhaltung von Umwelt und Natur.

Für die Integration der Leitmotive in technisch-ökonomisches Systemwissen werden Technosphären konstruiert. Basis für die Konstruktion bilden 22 für die Energiewende zentrale Richtungsentscheidungen in den Sektoren Strom, Wärme und Mobilität, die zuvor von einem interdisziplinären Projektteam identifiziert wurden (Bauknecht et al. 2018) und als Input-Parameter für die Technosphären dienen. In einem interdisziplinären Workshop mit ExpertInnen aus der Energiesystemanalyse sowie den Sozial- und Ingenieurwissenschaften werden mit der Cross-Impact-Bilanzanalyse (CIB) jeweils paarweise die gegenseitigen Beeinflussungen zwischen allen Richtungsentscheidungen bewertet, z. B. welchen Einfluss ein früherer Kohleausstieg auf die Struktur ,Stromerzeugung durch erneuerbare Energien“ haben kann. Die Einflüsse werden auf einer Skala von -2 (stark hemmend) bis +2 (stark fördernd) codiert. Abb. 1 zeigt schematisch den Prozess anhand von vier der 22 betrachteten Richtungsentscheidungen. Es ergibt sich ein komplexes Netz aus sich gegenseitig hemmenden und fördernden Richtungsentscheidungen. Mithilfe des CIB-Algorithmus werden 203 konsistente Technosphären, d. h. Bündel aus sich gegenseitig überwiegend fördernden Richtungsentscheidungen identifiziert (für mehr Details siehe Weimer-Jehle 2006).

\section{Zentrale Ergebnisse}

Ziel des Ansatzes ist es herauszufinden, welche Technosphären die identifizierten Leitmotive umsetzen und welche Leitmotive durch keine der konsistenten Technosphären realisiert werden können. Dazu bewertet das Projektteam mithilfe der CIB, wie vereinbar jede Richtungsentscheidung mit den in den Leitmotiven verfolgten Prinzipien ist. Alle Richtungsentscheidungen und Leitmotive wurden vor der Bewertung durch erklärende Essays beschrieben, um eine gemeinsame Bewertungsbasis zu gewährleisten. So wird ein Verbot fossiler Heizungen z. B. als wenig verträglich mit dem Leitmotiv „Gesellschaftliche Modernisierung “ bewertet $(-2)$, da dies ohne einen vorgeschalteten gesellschaftlichen Aushandlungsprozess als Eingriff in das für das Leitmotiv essenzielle partizipative Mitbestimmungsrecht einge- schätzt wird. Innerhalb einer Technosphäre entsprechen die einzelnen Richtungsentscheidungen einem Leitmotiv besser (grün), schlechter (orange) oder haben keinen Einfluss auf die Realisierung eines Leitmotivs (farblos). Tab. 2 zeigt dies an zwei verschiedenen Technosphären und dem Leitmotiv „Energiewende als Chance der gesellschaftlichen Modernisierung“. Insgesamt ergibt sich ein Score, mit dem bestimmt werden kann, welche der 203 Technosphären besonders konsistent oder inkonsistent mit den Leitmotiven sind. Von allen Technosphären unterstützen 146 dieses Leitmotiv (Range: +1 bis +12 ); 41 hemmen das Leitmotiv mehrheitlich (Range: -1 bis -8 ).

\section{Partizipative Storylines}

In ENSURE bringen Stakeholder ihre Positionen mithilfe von selbst entwickelten Storylines ein und begleiten über zwei Jahre deren Umsetzung in modellbasierte Energieszenarien. Die Storylines sollen konsistente, mögliche Entwicklungen in den rele- 


\begin{tabular}{|c|c|c|}
\hline \multirow[b]{2}{*}{ Richtungsentscheidungen } & \multicolumn{2}{|c|}{$\begin{array}{l}\text { Verträglichkeit des Leitmotivs „Energiewende als Chance der gesellschaftlichen Modernisierung“ } \\
\text { mit den zwei beispielhaften Technosphären (siehe Abb.1) }\end{array}$} \\
\hline & Technosphäre 1 (Score +12) & Technosphäre 2 (Score -8) \\
\hline A. Effizienz vs. EE-Nutzung & A2 Fokus auf erneuerbare Energien & A1 Fokus Effizienz/Dämmung \\
\hline B. Wärmenetze & B1 Ausbau Wärmenetze & B1 Ausbau Wärmenetze \\
\hline C. Heiztechnologie & C1 Verbot fossiler Heizungen & C1 Verbot fossiler Heizungen \\
\hline D. Power-to-Heat & D1 Starker P2H Einsatz & D1 Starker P2H Einsatz \\
\hline E. Emissionsreduktionsziele & E3 $100 \%$ Reduktion & E1 $80 \%$ Reduktion \\
\hline F. Strombedarf & F1 Steigend & F1 Steigend \\
\hline G. Fokus Deutschland/EU & G1 Fokus Deutschland & G2 Fokus EU \\
\hline H. Kohleausstieg & H1 Schneller Kohleausstieg & H2 Mittelfristiger Kohleausstieg \\
\hline I. Netzausbau vs. Alternativen & 12 Alternativen & 12 Alternativen \\
\hline J. Flexibilitätsoptionen & I3 Sektorkopplung & 15 Power-to-Gas \\
\hline K. Allgemeine Steuerungsformen & K3 Eigenverbrauch & K4 Zentrale Optimierung \\
\hline L. Antriebsarten & L2 Graduelle Elektrifizierung/Effizienzsteigerung & L3 Keine spezifische Förderung von Antriebstechnologien \\
\hline M. EE-Versorgung (Verkehr) & M1 Fokus EE-Strom im Verkehr & M3 Fokus alternative Kraftstoffe \\
\hline N. Sharing (Multimodalität) & N2 Sharing wird dominanter Lebensstil & N1 Sharing bleibt in der Nische \\
\hline O. Stadtplanung & O3 Starker Infrastrukturumbau ÖPNV/Rad & 01 MIV bleibt im Zentrum der Stadtplanung \\
\hline P. Rolle von Gasnetzen & P2 EE-Integration & P2 EE-Integration \\
\hline Q. Power-to-X & Q1 Fokus Direktstromnutzung & Q3 Fokus Methan \\
\hline R. Nutzung von Bioenergie & R1 Fokus Stromsektor & R1 Fokus Stromsektor \\
\hline S. Fossile KWK-Erzeugung & S1 KWK als kurzfristige Brückentechnologie & S2 KWK als mittelfristige Brückentechnologie \\
\hline T. Struktur der EE-Erzeugung & T3 Viel PV & T2 Offshore \\
\hline U. EE-Verteilung & U2 Dezentrale Verteilung & U1 Zentrale Verteilung \\
\hline V. Verkehrsvermeidung & V3 Maximale Verkehrsvermeidung & V1 Personen- und Güterverkehr steigt \\
\hline
\end{tabular}

Tab.2: Bewertung der Verträglichkeit zweier Technosphären mit einem Leitmotiv. Der Score ergibt sich aus der Addition der Punkte: dunkelorange $(-2)$, hellorange $(-1)$, dunkelgrün $(+2)$, hellgrün $(+1)$, farblos $(0)$.

vanten Sektoren bis 2030 beschreiben, den Raum der aus heutiger Sicht realistisch erscheinenden Entwicklungen aufspannen und die energiepolitischen Positionen der Stakeholder abdecken.

\section{Prozessbeschreibung}

Die Stakeholder werden in einem mehrstufigen Prozess ausgewählt. Zunächst wertet das Projektteam aktuelle energie- und klimapolitische Stellungnahmen von Verbänden und Organisationen aus unterschiedlichen gesellschaftlichen und wirtschaftlichen Bereichen aus und synthetisiert die Positionen zu 20 Kernfragen (Netzausbau, Klimaschutz, Sektorenkopplung, räumliche Verteilung Erneuerbare Energien, etc.). Darauf basierend werden elf Stakeholder identifiziert, die eine Bandbreite der Debatten widerspiegeln. Somit werden nicht nur Vertreter der Energiewirtschaft sowie Umwelt- und Naturschutzverbände eingeladen, sondern auch Arbeitnehmer- und Verbrauchervertreter sowie Vertreter von Kommunen und der Land- und Forstwirtschaft. In sieben Workshops treffen die Stakeholder wesentliche Entscheidungen zur Gestaltung der Storylines und legen Grundlagen für die Bewertung der Storylines und der hierauf aufbauenden Szenarien fest, siehe Abb. 2.

Die Aufgaben der WissenschafterInnen beschränken sich auf die Aufstellung des methodischen Rahmens und der Bereitstellung von Expertenwissen. Die Storylines werden mithilfe von 29 überwiegend qualitativen, das Energiesystem kennzeichnenden „Deskriptoren“ beschrieben; für die modellbasierten Szenarien werden sie in Abstimmung mit den Stakeholdern in quantitative Kenngrößen übersetzt und als Inputgrößen in die Modelle implementiert.

\section{Zentrale Ergebnisse}

Folgende vier Storylines werden entwickelt und dafür jeweils ein Szenario berechnet: 
Bildung der differenzierten Stakeholder-Gruppe

Gemeinsame Diskussion und Definition von Deskriptoren

Erster Entwurf der Storylines

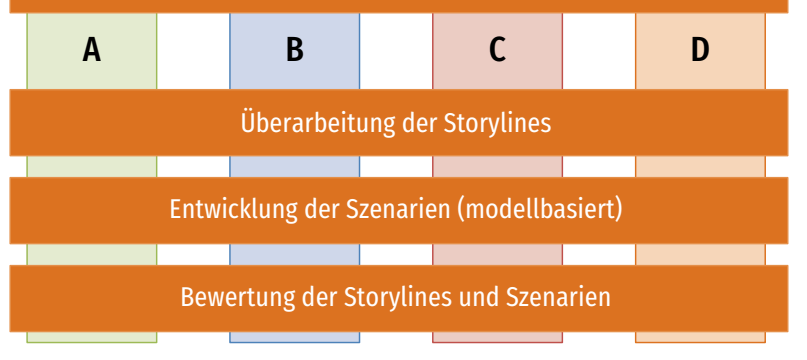

Abb.2: Arbeitsprozess zur Entwicklung der Storylines und Szenarien. Quelle: Eigene Darstellung

a) Referenzentwicklung;

b) Sehr ambitionierter Klimaschutz;

c) Stärker europäisch orientierte Energiewende mit ambitionierten Klimazielen;

d) Dezentrale Energiewende mit ambitionierten Klimazielen.

Storyline A geht von energiewirtschaftlichen Rahmendaten aus, wie sie im Netzentwicklungsplan 2030 (Szenario B, Version 2019) durch die Bundesnetzagentur festgelegt werden. Im Vergleich zu den anderen Storylines weist Storyline A das geringste klima-bezogene Ambitionsniveau auf. Die Treibhausgasemissionen würden hier bis 2030 um $55 \%$ gegenüber 1990 sinken. Das Ambitionsniveau der Storyline B zielt hingegen auf eine stringente Einhaltung des 2-Grad-Ziels. Die THG-Emissionen würden bis 2030 um $78 \%$ sinken. Storyline B bewegt sich damit jenseits der Grenze bisher ausgearbeiteter Energieszenarien und erfordert deutlichere System- und Verhaltensänderungen. Die Ambitionsniveaus der Storylines $\mathrm{C}$ und $\mathrm{D}$ entsprechen dem Mindestanspruch des Pariser Klimaschutzabkommens, THG-Emissionen würden in beiden Fällen um $67 \%$ sinken.

Auf Wunsch der Stakeholder werden Energieszenarien entwickelt, die weit über die Klimaschutzziele der Bundesregierung hinausgehen. Diese Diskussionen gehen von der Einbindung der deutschen Energiewende in die europäische Energie- und Klimapolitik sowie der Elektrifizierung von Wärme und Mobilität aus (Abb. 3). Zwar wird dem Klima- schutz eine herausragende Bedeutung beigemessen, in der Bewertung der Storylines durch die Stakeholder zeigt sich jedoch auch die hohe Relevanz sozio-ökonomischer und umweltbezogener Wirkungen der Szenarien.

\section{Diskussion}

Beide Ansätze liefern unterschiedliche Zugänge zu den zentralen Fragen des Beitrags. Der Leitmotiv-Ansatz entspringt der Annahme, dass Stakeholder-Positionen durch Weltbilder und Werte erklärt werden können. Leitmotive dienen dabei der Rekonstruktion tieferliegender Motive und bündeln Ziel- und Orientierungswissen gesellschaftlicher Akteure. Die methodische Herangehensweise kann eine Vielzahl von gesellschaftlichen Akteuren berücksichtigen, ohne dass die Analyse auf die Prozessteilnahme der Stakeholder angewiesen ist. Identifizierung und Ausgestaltung der Leitmotive basieren jedoch maßgeblich auf einer Interpretation des Forscherteams. Im Gegensatz dazu fokussiert der Storyline-Ansatz auf die Aushandlung von gemeinsam akzeptierten Richtungsentscheidungen, die eine Bandbreite gesellschaftlich erwünschter Energiezukünfte repräsentieren. In Anlehnung an Giesel (2007, S. 161) werden die Vorgehensweisen als analytisch-antizipativ versus diskursiv unterschieden. Ersteres wird in der Leitmotiv-Entwicklung genutzt, um die Implikationen identifizierter Leitmotive für die zukünftige Ausgestaltung des Energiesystems zu untersuchen. Leitmotive sind eine Art analytische Brille, mit der idealisierte „wenn-dann“-Analysen durchgespielt werden können: Welche Transformationspfade sind möglich, wenn sich das eine oder andere Leitmotiv in der Gesellschaft durchsetzt? Die Spiegelung von Leitmotiven an

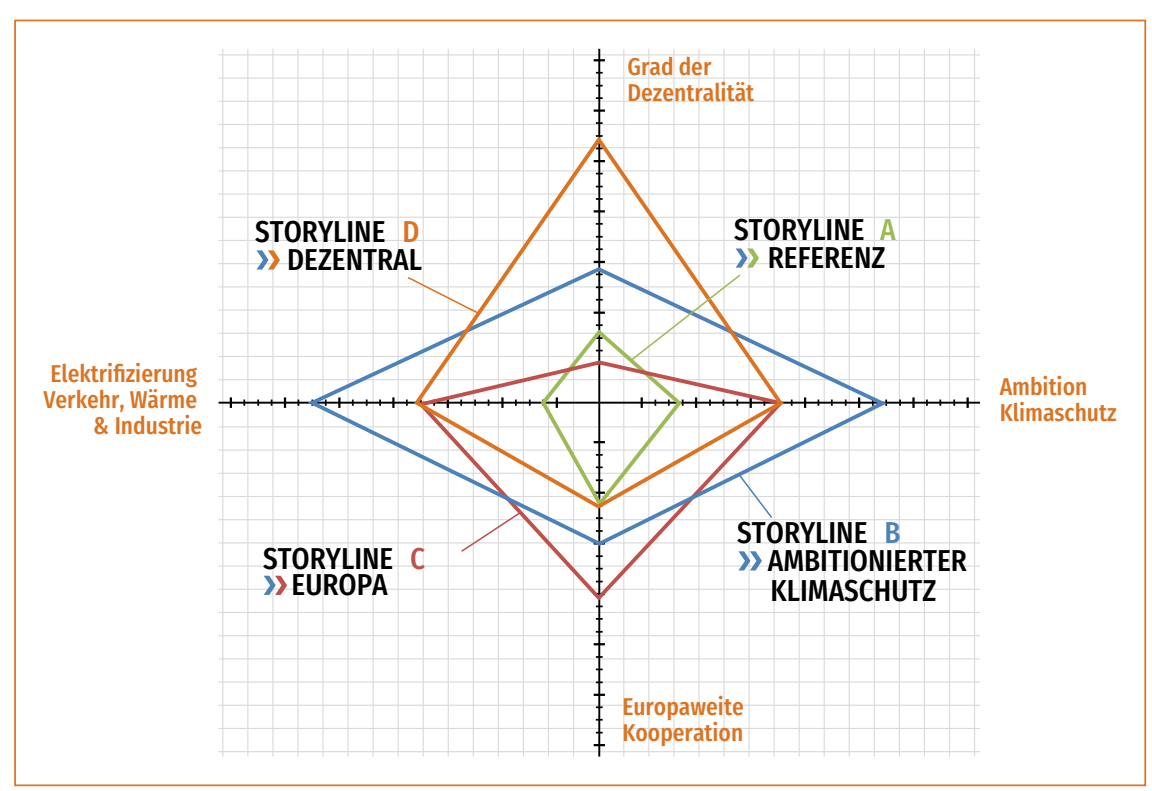

Abb.3: Charakteristische Merkmale der Storylines.

Quelle: Eigene Darstellung. Bildrechte: Schleswig-Holstein Netz AG 
einer großen Anzahl von Technosphären bietet die Möglichkeit, Stakeholder-Positionen und deren Einflüsse auf einen Transformationsprozess systematisch zu analysieren. Eine Herausforderung besteht darin, die Kompatibilitäten zwischen den technologisch-ökonomischen Richtungsentscheidungen und den Leitmotiven zu bewerten, da letztere in ihrer Natur qualitativ und vielschichtig sind. Die Nutzung der CIB schafft hier Transparenz, indem neben den Bewertungen auch qualitative Begründungen erfasst werden. Am Ende der Analyse stehen keine ,ready-to-use“ Ergebnisse; der methodische Ansatz bietet aber die Möglichkeit, gesellschaftliche Zukunftsvisionen und deren Implikationen für technisch-ökonomische Entscheidungsmöglichkeiten transparent aufzuarbeiten.

\section{Der methodische Ansatz ermöglicht}

\section{es, gesellschaftliche Zukunfts- visionen und deren Implikationen transparent aufzuarbeiten.}

Der diskursive Ansatz im Kontext der Storyline-Entwicklung ermöglicht eine Beteiligung ausgewählter Akteure in den Entwicklungsprozess von Szenarien (Timpe et al. 2018) und zielt auf eine Verknüpfung der Wissensbestände ab. Eine Gegenüberstellung wird nicht angestrebt, stattdessen werden iterative Modellierungsläufe genutzt, um Stakeholder-Positionen mit technischen Ausgestaltungsmöglichkeiten zu verknüpfen. Die Verknüpfung erfolgt durch die „Übersetzung“ qualitativer Deskriptoren in quantitative Kenngrößen. Für die Festlegung konkreter Zahlenwerte ist eine Verständigung darüber notwendig, welche Ausprägung die jeweiligen Deskriptoren annehmen dürfen, um kompatibel zu der ausgewählten Storyline zu sein. Ein ambitioniertes klimapolitisches Ziel wie in Storyline B impliziert z. B. einen relativ hohen Kohlenstoffdioxidpreis.

\section{Fazit}

Ergebnisse der diskursiven und analytisch-antizipativen Vorgehensweise können für die Ausgestaltung von Energiezukünften in unterschiedlicher Weise genutzt werden. Wird im Rahmen der Technikfolgenabschätzung davon ausgegangen, dass existierende Leitmotive zukünftige technische und ökonomische Entwicklungen beeinflussen, so hilft der Leitmotiv-Ansatz, mögliche Konsequenzen prospektiv zu erkennen und zu bewerten. Der Storyline-Ansatz zielt stärker auf energiepolitische Entscheidungen. Storylines und die daraus entwickelten Modellierungsergebnisse können direkt in der politischen und gesellschaftlichen Debatte aufgenommen werden. Positionen vom Projekt „externer“ Stakeholder können gegen die entwickelten Storylines gespiegelt und diskutiert werden. Diskurse über unterschiedliche gesellschaftliche Positionen können langfristig dazu beitragen, alternative Leitmotive herauszubilden und zu gesellschaftlich akzeptierten Richtungsentscheidungen zu formulieren (Canzler und Dierkes 2001) und dabei helfen, Leitmotive in partizipative Prozesse als zusätzliche Argumentationshilfen einzuspeisen. Beide hier präsentierten Ansätze schließen sich also keineswegs aus; sie eignen sich vielmehr als ergänzende Instrumente der Technikfolgenabschätzung und -gestaltung.

\section{Literatur}

Bauknecht, Dierk et al. (2018): Pfadentscheidungen der Energiewende. Ein Bericht im Rahmen des Kopernikus-Projekts ENavi. Potsdam: IASS. Online verfügbar unter https://www.oeko.de/fileadmin/oekodoc/ Pfadentscheidungen-Energiewende.pdf, zuletzt geprüft am 18. 10.2019. Becker, Egon; Jahn, Thomas (1999): Sustainability and the social sciences. A cross-disciplinary approach to integrating environmental considerations into theoretical reorientation. London: Zed Books.

Berkhout, Frans (2006): Normative expectations in systems innovation. In: Technology Analysis \& Strategic Management 18 (3-4), S. 299-311. BMBF - Bundesministerium für Bildung und Forschung (2019): Kopernikus Projekte. Die Zukunft unserer Energie. Online verfügbar unter https://www.kopernikus-projekte.de/, zuletzt geprüft am 27.06.2019. Canzler, Weert; Dierkes, Meinolf (2001): Informationelle Techniksteuerung Öffentliche Diskurse und Leitbildentwicklungen. In: Politische Vierteljahres schrift, Sonderheft 31 „Politik und Technik. Analysen zum Verhältnis von technologischem, politischem und staatlichem Wandel am Anfang des 21. Jahrhunderts" hg. von Georg Simonis und Thomas Saretzki, S. 457-475. Cuppen, Eefje; Bosch-Rekvelt, Marian; Pikaar, Ewout; Mehos, Donna (2016): Stakeholder engagement in large-scale energy infrastructure projects. Revealing perspectives using Q methodology. In: International Journal of Projekt Management 34, S. 1347-1359.

de Haan, Gerhard (2001): Die Leitbildanalyse. Ein Instrument zur Erfassung zukunftsbezogener Orientierungsmuster. In: Gerhard de Haan, Ernst-Dieter Lantermann, Volker Linneweber und Fritz Reusswig (Hg.): Typenbildung in der sozialwissenschaftlichen Umweltforschung. Wiesbaden: Springer vs. Giesel, Katharina (2007): Leitbilder in den Sozialwissenschaften. Begriffe,

Theorien und Forschungskonzepte. Wiesbaden: vs Verlag für Sozialwissenschaften.

Gleich, Arnim von; Brand, Urte; Stührmann, Sönke; Gößling-Reisemann, Stefan; Lutz-Kunisch, Birgit (2010): Leitorientierte Technologie- und Systemgestaltung. In: Klaus Fichter, Arnim von Gleich, Reinhard Pfriem und Bernd Siebenhüner ( $\mathrm{Hg}$.): Theoretische Grundlagen für erfolgreiche Klimaanpassungsstrategien. Bremen: Projektkonsortium nordwest2050.

Heyen, Dirk; Brohmann, Bettina; Libbe, Jens; Riechel, Robert; Trapp, Jan (2018) Stand der Transformationsforschung unter besonderer Berücksichtigung der kommunalen Ebene. Berlin: Öko-Institut e. V.

Mielke, Jahel; Vermeßen, Hannah; Ellenbeck, Saskia; Fernandez Milan, Blanca (2016): Stakeholder involvement in sustainability science. A critical review. In: Energy Research \& Social Science 17, S. 71-81.

Nakićenović, Nebojsa (2000): Greenhouse gas emission scenarios. In: Technological Forecasting \& Social Change 65 (2), S. 149-166.

Rosenstiel, Lutz von (2007): Grundlagen der Organisationspsychologie. Stuttgart: Schäffer-Poeschel Verlag. 
Timpe, Christoph; Bauknecht, Dierk; Flachsbarth, Franziska; Koch, Matthias (2018): Transparenz Stromnetze. Stakeholder-Dialog und Modellierung zum Netzausbau und Alternativen. Berlin: Öko-Institut e.V.

Trutnevyte, Evelina; Barton, John; O'Grady, Aine; Ogunkunle, Damiete; Pudjianto, Danny; Robertson, Elisabeth (2014): Linking a storyline with multiple models. A cross-scale study of the UK power system transition. In: Technological Forecasting \& Social Change 89, S.26-42.

Weimer-Jehle, Wolfgang; Prehofer, Sigrid; Vögele, Stefan (2013): Kontextszenarien. Ein Konzept zur Behandlung von Kontextunsicherheit und Kontextkomplexität bei der Entwicklung von Energieszenarien. In: TATuP - Zeitschrift für Technikfolgenabschätzung in Theorie und Praxis 22 (2), S. 27-35. DOI: 10.14512/tatup.22.2.27.

Weimer-Jehle, Wolfgang (2006): Cross-impact balances. A system-theoretical approach to cross-impact analysis. In: Technological Forecasting \& Social Change 73 (4), S. 334-361.

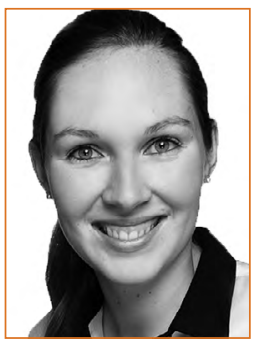

\section{RICARDA SCHMIDT-SCHEELE}

forscht am ZIRIUS zu Szenarien im Kontext von Energiewende und Nachhaltigkeit. Im Fokus ihrer Arbeit stehen Methoden der Erstellung, Wahrnehmung und Verarbeitung von Zukunftsperspektiven durch gesellschaftliche Akteure.

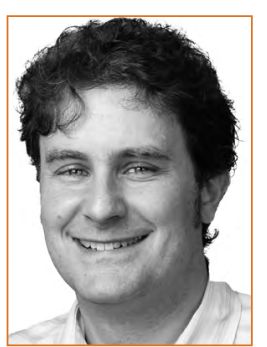

\section{DR. DIERK BAUKNECHT}

ist Senior Researcher im Bereich Energie \& Klimaschutz am Öko-Institut. Promotion an der University of Sussex mit einer Arbeit zu Stromnetztransformation. Er arbeitet zur Transformation des Stromsystems aus der Perspektive der Energiesystemanalyse als auch der Regulierung.
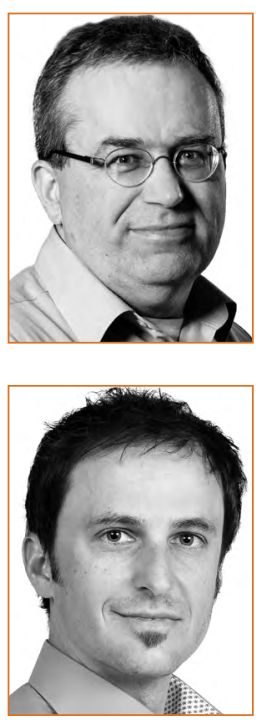

\section{DR. WITOLD-ROGER POGANIETZ}

ist promovierter Volkswirt und arbeitet am ITAS. Forschungsschwerpunkte sind die Analyse von sozio-technischen Treibern und Hemmnissen bei der Transformation von Energiesystemen in Europa und des "Globalen Südens".

\section{DOMINIK SEEBACH}

ist Geoökologe und Senior Researcher im Bereich Energie \& Klimaschutz am Öko-Institut. Er arbeitet zur Analyse politischer und ökonomischer Instrumente im Bereich der nachhaltigen Stromerzeugung und -nutzung.

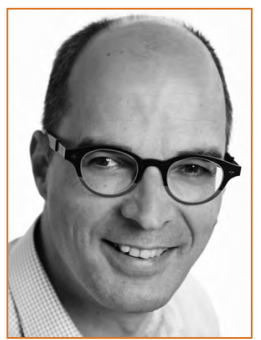

\section{CHRISTOF TIMPE}

ist Diplom-Ingenieur Energietechnik und Leiter des Bereichs Energie \& Klimaschutz am Öko-Institut. Er arbeitet zu nationalen und europäischen Strategien zur Entwicklung erneuerbarer Energien und zur nachhaltigen Transformation des Stromsystems in Deutschland und Europa.

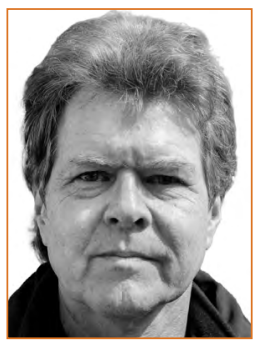

\section{DR. WOLFGANG WEIMER-JEHLE}

ist Physiker und forscht am ZIRIUS auf den Gebieten Energie, Nachhaltigkeit, Szenariomethodik und Methodenforschung zur qualitativen Systemanalyse. Schwerpunkt ist die Methodenforschung zur Cross-Impact-Bilanzanalyse, die von ihm entwickelt wurde.

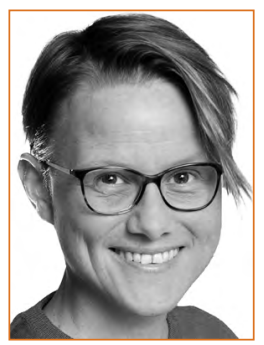

\section{DR. ANNIKA WEISS}

forscht als Ingenieurin am ITAS zu Energiesystemen. Schwerpunkt ist die Verknüpfung gesellschaftlicher und technischer Entwicklungen in Szenarien.

2016 erfolgte die Promotion zum Thema Energiebilanz von Mikroalgentreibstoff. Weitere Forschungsfelder sind Wasser und nachhaltige Transformation. 